\title{
In memory of Piero Sensi (1920-2013)
}

\author{
The Journal of Antibiotics (2014) 67, 609-611; doi:10.1038/ja.2014.104
}

A scientist of worldwide reputation (Figure 1), Piero Sensi is known mainly for the discovery of rifampicin, a fundamental contribution to the therapy of tuberculosis. Born in 1920 near Viterbo, a town in central Italy, he attended high school in Naples and then enrolled at the 'Università di Napoli Federico II', graduating in 1944 with a thesis on physical chemistry. In 1959 he earned the academic title of 'Libero Docente' in Pharmaceutical and Toxicological Chemistry.

His first work experience involved the synthesis of new dyes in a laboratory of Montecatini, a chemical company. In 1950, he joined the pharmaceutical company Lepetit with the goal of establishing a laboratory of physical chemistry. Within a short time he had organized a laboratory equipped with the most up-to-date spectroscopic instruments, operated by a few young chemists who, under his guidance, rapidly became experts in instrumental analysis. In the early fifties, Lepetit was entering the antibiotic business, focusing on process development. The physical chemistry laboratory proved essential in the study and improvement of fermentation and downstream processing. These efforts resulted in a complete industrial process for the production of tetracycline that proved highly competitive in the world market. Additionally, by varying the fermentation process, Sensi produced the new antibiotic bromotetracycline. This encouraged Lepetit to start a program of screening for new antibiotics.

In the mid-fifties, the new Department of Antibiotics and Natural Products, of which Sensi was shortly named Director, was established. The Department comprised a small group of young motivated scientists, who rapidly gained experience in the various disciplines involved in a screening program. In late 1957 an actinomycete strain was found, classified as Streptomyces mediterranei (later reclassified as Amycolatopsis mediterranei), which appeared novel and produced interesting active substances. A crude extract of the fermentation broth had strong inhibitory activity against Gram-positive bacteria and also had a protective effect in murine models of infection. Paper chromatography revealed that the antibiotic was composed of five substances, which were named rifamycins A, B, C, D and E. Components A, C, D and E were chemically quite similar: C and D appeared to have much greater biological activity than the others, but, when injected into mice, they caused severe local lesions. For this reason, rifamycin $\mathrm{B}$, which had at first been overlooked due to its poor activity, was considered for further studies. This decision was supported by the unexpected finding that adding barbital to the culture medium produced profound alterations in the composition of the rifamycin complex: A, C, D and E were nearly absent, whereas rifamycin $\mathrm{B}$ production was increased fivefold.
How Sensi succeeded in transforming an antibiotic with modest properties into the progenitor of a family of hundreds of semisynthetic antibiotics, five of which have been approved for clinical use, is a complex, fascinating story that can only be summarized here. The surprising observation that rifamycin B in aqueous solution was slowly transformed into substances with greater antimicrobial potency confirmed Sensi's intuition that rifamycins could be improved by chemical transformation. He correctly deduced that the spontaneous transformation in aqueous solution could only be the result of an oxidation, a hydrolysis, or both, in sequence. Indeed, he was able, within a short time, to reproduce the hypothesized reactions, obtaining, by oxidation followed by mild hydrolysis, two novel products, rifamycins $\mathrm{O}$ and $\mathrm{S}$. The molecules were both very active in vitro, but not in experimental infections, most likely because of their insolubility in water. This might have been the end of the story, since none of the seven rifamycins tested up to that point of time appeared to have potential for therapeutic development. However, because the UV spectrum of rifamycin $S$ indicated the presence of a quinone, Sensi reasoned that with a mild reduction reaction it could be transformed into a more soluble hydroquinone. Addition of ascorbic acid to a solution of rifamycin $S$ indeed yielded a new reduced soluble product, named Rifamycin SV, which was effective in experimental infections in mice. Its therapeutic efficacy by parenteral administration was confirmed by extensive clinical investigations. The sodium salt of rifamycin SV was marketed in 1963 as an anti-Grampositive bacterial agent in several European and South American countries under the name of 'Rifocin'.

Rifamycin SV was produced in 1961, and in 1962 Sensi initiated an ambitious project of rational modifications of the rifamycins. The main objective was to obtain a product with a broad antibacterial spectrum that was orally active and effective in the therapy of tuberculosis. The approach involved modifying the molecules' physical properties such as lipophilicity, acidity, etc., which might affect oral absorption, pharmacokinetics or metabolism, without interfering with the antibacterial activity. Rifamycins $\mathrm{O}$ and $\mathrm{S}$, in addition to rifamycin $\mathrm{B}$, appeared to be excellent starting materials for transformation and gave rise to several series of active products. In 1963 Professor Vladimir Prelog at ETH in Zurich succeeded in elucidating the structure of rifamycin B by chemical degradation and extensive use of ${ }^{1} \mathrm{H}$ NMR. This gave a new impetus to the synthesis program, since knowledge of the structure greatly facilitated the identification of which sites of the molecule were essential for activity and which could be safely modified. In all, about two hundred products were synthesized, their biological activities progressively approaching the goal of a broad-spectrum orally active drug. The final result of the 


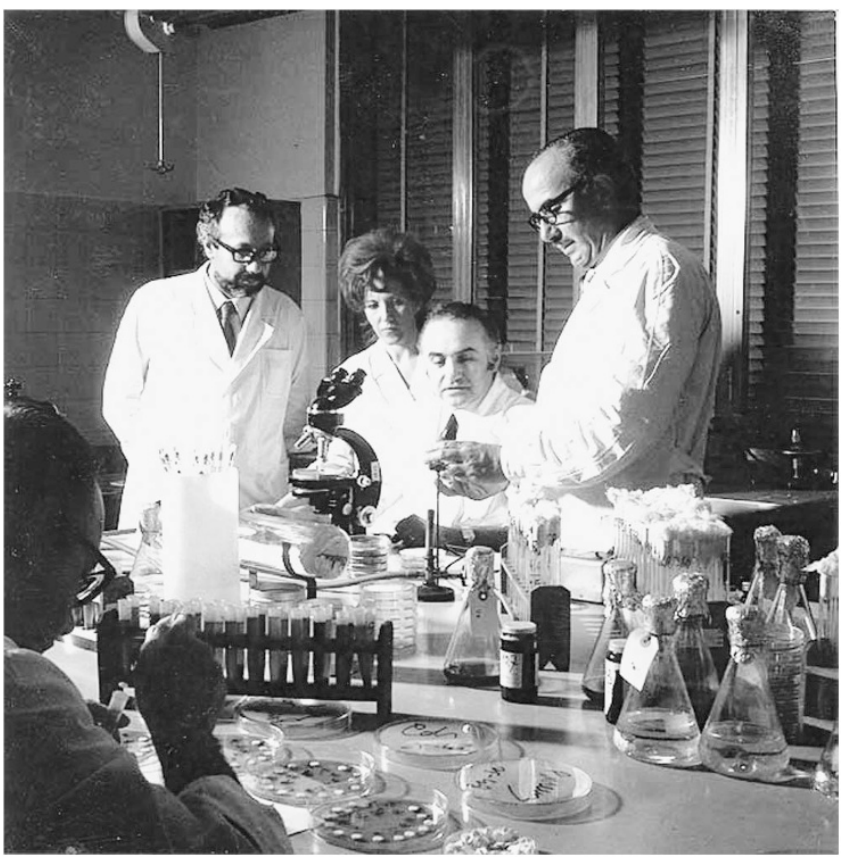

Figure 1 Prof Sensi and his colleagues. It was taken in the early seventies, during one of his usual visits to the laboratory to discuss the latest results of screening. From left to right, Dr Giancarlo Lancini, Ms Grazia Beretta, Mr Ermes Pagani and Prof Piero Sensi. A full color version of this figure is available at The Journal of Antibiotics journal online.

project was rifampicin - a product with a therapeutic efficacy well beyond initial expectations.

Although the chemists and microbiologists of the department were focusing on the synthesis and evaluation of rifamycins, screening for new antibiotics continued. To avoid the high rate of rediscovery of known products, some innovations in the screening process were introduced. A program to isolate Thermoactinomyces strains soon yielded a new antibiotic, thermorubin, which was interesting because of its broad spectrum of antibacterial activity and its novel mechanism of action, but it was not effective in animal infection models. The addition of a test for antiviral activity to the traditional antibacterial panel led to the isolation of a product with moderate antiviral activity, but interesting antitumor activity in vitro. This product, named alanosine, was studied at the US National Cancer Institute, NIH, in Bethesda for its possible use against head and neck tumors, but was abandoned after phase 1 clinical studies.

In 1966 Sensi was named Director of the Lepetit Research Laboratories, which included, besides the Department of Antibiotics and Natural Products, the Departments of Organic Synthesis, Pharmacology, Toxicology, Medical Microbiology and Physical Chemistry. His main goal was to implement a new organization of the Laboratory to facilitate communication among scientists in the various disciplines. The resulting enhanced collaboration contributed to the development of higher-quality projects and a general improvement in the Laboratory's performance.

Although the new position was very demanding in terms of the amount of time dedicated to management, Sensi continued to guide the scientific activity of the laboratory, directly supervising the various lines of research in the Department of Antibiotics. Synthesis of rifamycin analogs continued, and a derivative named rifapentine was found to be about as active as rifampicin but with a different pharmacokinetic profile, permitting twice weekly, rather than daily, administration. After the initial clinical trials conducted by Lepetit, its development was completed by Hoechst and it is marketed under the trade name Priftin.

During this period, Sensi also became interested in the biology of A. mediterranei and in the rifamycin biosynthetic process. A study of the last step of the process demonstrated that rifamycin SV was the immediate precursor of rifamycin B. This finding suggested it would be possible to directly produce rifamycin SV by fermentation, rather than by the usual chemical degradation of rifamycin B. Indeed, by mutagenesis a strain was obtained that produced rifamycin SV in good yields. Today, most industrial fermentation plants around the world use the rifamycin SV-producing strain for the manufacture of rifampicin and other rifamycin derivatives.

Initial attempts to elucidate the biosynthetic pathway of rifamycins, notably in a joint study by the Organic Chemistry Laboratory of ETH and the Lepetit Research Laboratory, using ${ }^{14} \mathrm{C}$ - or ${ }^{1} \mathrm{H}$-labeled propionate, gave ambiguous results. Therefore, Sensi's collaborators turned to ${ }^{13} \mathrm{C}$ NMR spectroscopy, a technique just proposed for studying biosynthetic pathways. This attempt was completely successful, demonstrating that the origin of the molecule was a single polyketide chain, which was also the origin of a number of other natural ansamycins.

In the mid-sixties the scientists involved in the antibiotic screening program became concerned by the perception that, although the number of new antibiotics discovered annually was increasing, the number that appeared to be potentially therapeutically useful was steadily dwindling. At Lepetit, two important new approaches to combat this trend were devised and successfully implemented. A simple method was found for the mass isolation of strains of Actinoplanes, then a little known genus of actinomycetes. In one year almost ten thousand strains were isolated and a few novel products identified, among which was the broad-spectrum antibiotic purpuromycin. One year later a novel inhibitor of RNA polymerase was isolated from cultures of Actinoplanes deccanensis. The product denoted as lipiarmycin (from 'leap year', the producing strain was isolated on 29 February 1972) was later named lipiarmycin A, when it was realized that it is a complex of two minor factors and two major factors named lipiarmycin A3 and A4. The antibiotic was the first example of a new class of macrolides that inhibit transcription initiation. Lipiarmycin had modest activity against Gram-positive bacteria, but excellent activity against certain anaerobes. At the time the potential importance of this observation was not evident, and the product was abandoned. Thirty years later lipiarmycin A3, with the name fidaxomicin, was developed by Optimer Pharmaceuticals and approved by the FDA for the treatment of Clostridium difficileassociated diarrhea.

At this point, it became clear that the bottleneck in the Actinoplanes screening program was no longer finding cultures that were active in the initial tests, but the number of culture extracts that could be examined for novelty and antimicrobial properties. To increase the efficiency of the screening, a simple assay was devised to select the cultures that contained cell wall inhibitors. The assay consisted of determining in parallel the antibacterial activity of broth filtrates on a Staphylococcus aureus strain and its isogenic L-form. A large difference (less or no activity against the L-form) was considered evidence of the presence of an inhibitor of cell wall biosynthesis in the culture. The combination of screening Actinoplanes strains and early identification of cell wall formation inhibitors led in the early seventies to the discovery of three interesting antibiotics: gardimycin, ramoplanin and teichomycins. Gardimycin, a narrow spectrum antibiotic, was the first known example of a new group of lantibiotics characterized by their 
small size and their mechanism of action. It attracted the interest of several research groups because of the surprising finding that it was more effective in curing experimental infections than could be predicted from its in vitro activity. This unusual property is also shown by several of its semisynthetic derivatives. Ramoplanin is a lipopeptide noteworthy for its excellent activity against multi-resistant Gram-positive bacteria. Although active upon parenteral administration in experimental infections, it was not developed because of poor tolerability. It has been approved for a phase III clinical study against C. difficile. Teichomycins are a family of glycopeptide antibiotics, of which teicoplanin (formerly known as teichomycin $\mathrm{A}_{2}$ ) became, after rifampicin, the most important therapeutic agent discovered and developed at Lepetit. It has a similar spectrum of activity and the same mechanism of action as vancomycin; however, it has better pharmacokinetic properties, as it is therapeutically effective with a single daily i.v. administration, in contrast to vancomycin, which at that time had to be administered i.v. four times daily.

In 1974 Sensi was named Central Director of Lepetit Research and Development, an institution employing about 150 university graduates, and therefore he could no longer directly guide the complex chemical and biological studies required for antibiotic development. However, he never ceased to advise and encourage his former collaborators. At the same time, he continued his scientific activity by giving lectures and contributing to important treatises on medicinal chemistry. Upon his retirement in 1978 he was appointed Professor of Industrial Microbiology at the University of Milano.

Piero Sensi was a pleasant person to work with. He had a relaxed attitude toward the events of life and usually would comment upon a success with an understatement. He was never discouraged by a setback and patiently went on to overcome difficulties. He had a positive attitude toward his collaborators, and indeed they never felt judged but always felt understood and appreciated. He used to informally discuss with them the results and advancement of the research projects, encouraging the proposal of new ideas so that everybody felt involved in the progress of research. When he became Director of Research Laboratories he instituted participation of all professionals in the decision-making process for the research programs. He introduced a system of interdisciplinary teams across the Departments, each team corresponding to a research target area (antihypertensives, central nervous system, etc.), comprising all the chemists and biologists working in that area. The team had the responsibility of analyzing the progress and results of the projects under way and of assessing the scientific value and probability of success of new project proposals. As a rule, decisions of the teams were based on consensus.

Sensi published over a hundred scientific papers, reviews and chapters of important treatises. He was often invited to deliver lectures at congresses and symposia. His personal and scientific qualities were widely appreciated, especially by scientists working in various aspects of antibiotic research. He received significant recognitions, including the Gold Medal of the President of the (Italian) Republic for Public Health Merit (1983), the 'Giuseppe Borgia' award of the National Academy of the Lincei (1976), the Gold Medal for Physical Sciences of the Accademia Nazionale delle Scienze (1989) and the 'Hamao Umezawa Award' of the International Society of Chemotherapy (1985). Additionally, a 'Laurea Honoris Causa' was conferred on him by the University of Bologna in 1991. Finally, what may be less-widely known publicly is Sensi's great merit for having raised a generation of young researchers into fine scientists who contributed in making the Lepetit Research Laboratory a center of scientific excellence. They now mourn over Piero Sensi-a scientist, teacher and friend.

Giancarlo Lancini

Screening, Foundation FIIRV, Gerenzano, Italy E-mail: gclan@libero.it 